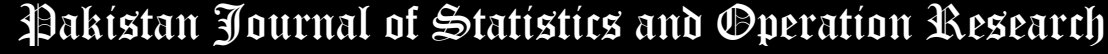

\section{The Negative Binomial - Weighted Garima Distribution: Model, Properties and Applications}

\author{
Winai Bodhisuwan ${ }^{1}$, Pornpop Saengthong ${ }^{2 *}$
}

* Corresponding Author

1. Department of Statistics, Faculty of Science, Kasetsart University, Chatuchak, Bangkok, 10900 Thailand fsciwnb@ku.ac.th

2. Institute for Research and Academic Services, Assumption University, Bang Sao Thong, Samuthprakarn, 10570 Thailand

pornpopsng@au.edu

\begin{abstract}
In this paper, a new mixed negative binomial (NB) distribution named as the negative binomial-weighted Garima (NB-WG) distribution is introduced for modeling count data. Two special cases of the formulation distribution, including negative binomial- Garima (NB-G), and negative binomial-size biased Garima (NB-SBG), are obtained by setting a specified parameter. Some statistical properties, such as the factorial moments, the first four moments, variance, skewness and kurtosis, have also been derived. Parameter estimation is done using maximum likelihood estimation (MLE), and real data sets are discussed to demonstrate the usefulness and applicability of the proposed distribution.
\end{abstract}

Key Words: Mixed negative binomial distribution, weighted Garima distribution, negative binomial-weighted Garima distribution, overdispersion, count data

\section{Introduction}

In the past decades, there has been much research done on the development of discrete distributions for analyzing and modeling count data. An original approach for analyzing count data is the Poisson distribution. However, the main condition of the Poisson distribution is the equal dispersion of the count data, which rarely happens in real-life applications. Count data often display over-dispersion (the variance is greater than the mean), leading to alternative modeling, which is more flexible than the Poisson distribution. The negative binomial (NB) distribution, a mixture of the Poisson and gamma distributions, is another popular model for count data. It includes two parameters that introduce substantial flexibility into the modeling of the variance function, and thus heteroskedasticity (Rainer, 2008). Although the NB distribution allows for over-dispersion, it does not take care of extra zeros in the data. Many applications for modeling count data with extra zeros have indicated that traditional statistical distributions such as the Poisson and NB distributions cannot be used efficiently. The Poisson distribution tends to under-estimate the number of zeros given the mean of the data, whereas the NB may over-estimate zeros, but under-estimate observations with a count (Lord and Geedipally, 2011).

Count data with overdispersion and extra zeros are often solved by mixing the Poisson or NB distribution with a lifetime distribution. Many studies have shown that mixed and compound distributions such as the Poisson-inverse Gaussian distribution (Willmot, 1987), NB-inverse Gaussian distribution (Gómez-Déniz et al., 2008), NB-lindley distribution (Zamani and Ismail, 2010), NB-beta exponential distribution (Pudprommarat et al., 2012), NB-erlang distribution (Kongrod et al., 2014), and NB-Sushila distribution (Yamrubboon et al., 2017) are more suitable for the count data than the Poisson and NB distributions. Therefore, in order to obtain another competitive alternative mixed distribution to the aforementioned distributions, we looked for a new lifetime distribution that can be expressed in closed form for building such a mixed model. The weighted Garima (WG) distribution is a new lifetime distribution 
that includes two-parameters proposed by Eyob and Shanker (2018). The goodness of fit of the WG distribution has been examined with a sample of real lifetime data; it has been shown a better fit compared to the exponential, Lindley, Garima, Gompertz, generalized exponential, lognormal and weighted Lindley distributions. As a result, a new mixed NB distribution named as the Negative Binomial-Weighted Garima (NB-WG) Distribution, which is obtained by mixing the NB distribution with a WG distribution, is proposed to model count data. This new distribution has a heavy tail, seems to be positively skewed and may be considered as a preferable alternative for modeling overdispersed count data.

The paper is organized as follows. In Section 2, we present the components for developing the NB-WG distribution. In addition, the NB-WG distribution and its special cases, along with their probability mass functions (pmf) are derived. Some properties of the NB-WG distribution, and parameter estimation are provided in Sections 3 and 4 , respectively. Section 5 compares the performance between the Poisson, NB, and NB-WG distributions using goodness of fit. Finally, the conclusions of the paper are presented in the last section.

\section{Construction of the NB-WG Distribution}

This section explains how to develop the NB-WG distribution. A mixture of the negative binomial (NB) and weighted Garima (WG) distributions is discussed in detail. We begin by showing the NB distribution and some of its properties.

The probability mass function (pmf) of the NB distribution with parameters $r>0$ and $0<p<1$ can be written as

$$
f(x ; r, p)=\left(\begin{array}{c}
r+x-1 \\
x
\end{array}\right) p^{r}(1-p)^{x}, \quad x=0,1,2, \ldots
$$

In Equation (1), if $X$ has a NB distribution, its first two moments about zero and the factorial moment (Balakrishnan and Nevzorov, 2003) are given, respectively, by

$$
\begin{aligned}
& E(X)=\frac{r(1-p)}{p}, \\
& E\left(X^{2}\right)=\frac{r(1-p)[1+r(1-p)]}{p^{2}}, \\
& \mu_{[k]}(X)=E[X(X-1) \ldots(X-k+1)] \\
& =\frac{\Gamma(r+k)}{\Gamma(r)} \frac{(1-p)^{k}}{p^{k}}, \quad k=1,2, \ldots
\end{aligned}
$$

where $\Gamma($.$) is the complete gamma function defined by$

$$
\Gamma(t)=\int_{0}^{\infty} x^{t-1} e^{-x} d x, \quad t>0 .
$$

Eyob and Shanker (2018) developed the two parameter WG distribution with the probability density function (pdf)

$$
f(x ; \theta, \beta)=\frac{\theta^{\beta}}{(\theta+\beta+1)} \frac{x^{\beta-1}}{\Gamma(\beta)}(1+\theta+\theta x) e^{-\theta x} \quad ; x>0, \theta>0, \beta>0
$$

where $\Gamma(\beta)=\int_{0}^{\infty} e^{-y} y^{\beta-1} d y, \quad y>0, \beta>0$.

The moment generating function (mgf) of the WG distribution is given by

$$
M_{X}(t)=\frac{\theta^{\beta}}{(\theta+\beta+1)(\theta-t)^{\beta+1} \Gamma(\beta)}[(\theta+1)(\theta-t) \Gamma(\beta)+\theta \Gamma(\beta+1)] .
$$

Based on the above information, the concept of the NB-WG distribution is defined as follows.

Definition. Let $X$ be a random variable of the $N B-W G(r, \theta, \beta)$ distribution where $X$ has the NB distribution with parameter $r>0$ and $p=\exp (-\lambda)$ when $\lambda$ is distributed as the WG distribution with positive parameters $\theta$ and $\beta$, i.e., $X \mid \lambda \sim N B(r, p=\exp (-\lambda))$ and $\lambda \sim W G(\theta, \beta)$ for $r, \theta, \beta>0$.

Theorem 1. Let $X \sim N B-W G(r, \theta, \beta)$. The pmf of $\mathrm{X}$ is given by

$$
f(x ; r, \theta, \beta)=\frac{\theta^{\beta}}{(\theta+\beta+1) \Gamma(\beta)}\left(\begin{array}{c}
r+x-1 \\
x
\end{array}\right) \sum_{j=0}^{x}\left(\begin{array}{c}
x \\
j
\end{array}\right)(-1)^{j} \frac{(\theta+1)(\theta+r+j) \Gamma(\beta)+\theta \Gamma(\beta+1)}{(\theta+r+j)^{\beta+1}},
$$

where $\mathrm{x}=0,1,2, \ldots$, for $r, \theta$ and $\beta>0$. 
Proof. If $X \mid \lambda \sim N B(r, p=\exp (-\lambda))$ in (1) and $\lambda \sim W G(\theta, \beta)$ in (3), then the pmf of $X$ can be derived from

$$
f(x ; r, \theta, \beta)=\int_{0}^{\infty} h(x \mid \lambda) g(\lambda ; \theta, \beta) d \lambda,
$$

where

$$
h(x \mid \lambda)=\left(\begin{array}{c}
r+x-1 \\
x
\end{array}\right) e^{-\lambda r}\left(1-e^{-\lambda}\right)^{x}=\left(\begin{array}{c}
r+x-1 \\
x
\end{array}\right) \sum_{j=0}^{x}\left(\begin{array}{l}
x \\
j
\end{array}\right)(-1)^{j} e^{-\lambda(r+j)} .
$$

By substituting (7) into (6), we have

$$
\begin{aligned}
f(x ; r, \theta, \beta) & =\left(\begin{array}{c}
r+x-1 \\
x
\end{array}\right) \sum_{j=0}^{x}\left(\begin{array}{l}
x \\
j
\end{array}\right)(-1)^{j} \int_{0}^{\infty} e^{-\lambda(r+j)} g(\lambda ; \theta, \beta) d \lambda \\
& =\left(\begin{array}{c}
r+x-1 \\
x
\end{array}\right) \sum_{j=0}^{x}\left(\begin{array}{l}
x \\
j
\end{array}\right)(-1)^{j} M_{\lambda}(-(r+j)) .
\end{aligned}
$$

It can be easily verified that the pmf of the NB-WG $(r, \theta, \beta)$ is obtained by replacing the mgf of the WG distribution in (4) with $t=-(r+j)$ into (8). Then, it is finally given as

$$
\begin{aligned}
f(x ; r, \theta, \beta) & =\frac{\theta^{\beta}}{(\theta+\beta+1) \Gamma(\beta)}\left(\begin{array}{c}
r+x-1 \\
x
\end{array}\right) \\
& \times \sum_{j=0}^{x}\left(\begin{array}{l}
x \\
j
\end{array}\right)(-1)^{j} \frac{(\theta+1)(\theta+r+j) \Gamma(\beta)+\theta \Gamma(\beta+1)}{(\theta+r+j)^{\beta+1}} .
\end{aligned}
$$

The pmf of the NB-WG distribution with different values of parameters is plotted in Figure 1. Special cases of the NBWG distribution include the negative binomial-Garima distribution $(\beta=1)$ and the negative binomial-size biased Garima distribution $(\beta=2)$. The proof then follows directly from Corollary 1.1 and 1.2, respectively.
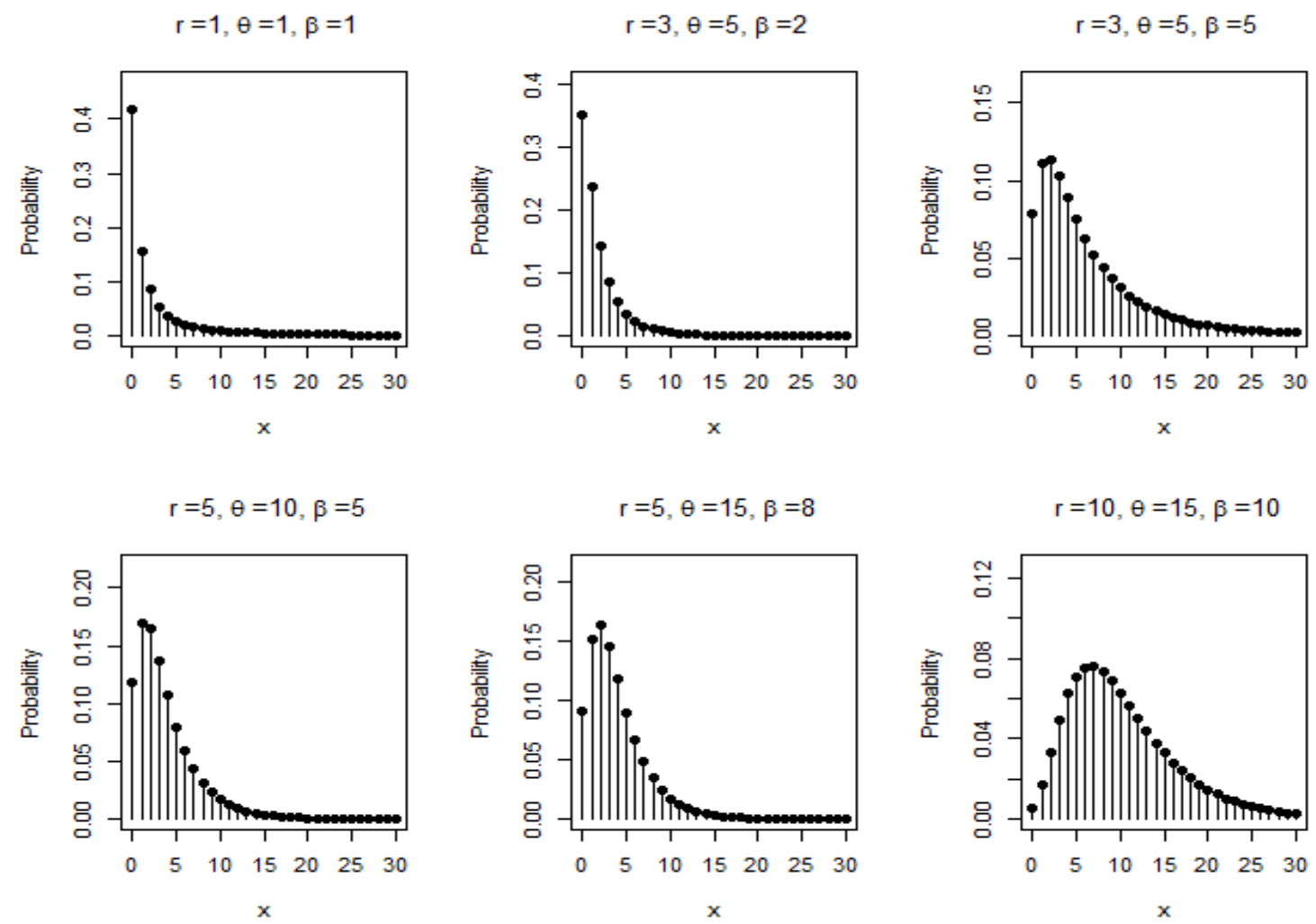

Figure 1: The probability mass function of a NB-WG random variable with some specific values of $\boldsymbol{r}, \boldsymbol{\theta}$ and $\boldsymbol{\beta}$ 
Corollary 1.1. If $\beta=1$, then the NB-WG distribution reduces to the negative binomial-Garima (NB-G) distribution with the pmf given by

$$
f(x ; r, \theta)=\frac{\theta}{(\theta+2)}\left(\begin{array}{c}
r+x-1 \\
x
\end{array}\right) \sum_{j=0}^{x}\left(\begin{array}{l}
x \\
j
\end{array}\right)(-1)^{j} \frac{\theta+(\theta+1)(\theta+r+j)}{(\theta+r+j)^{2}}
$$

where $\mathrm{x}=0,1,2, \ldots$, for $r$ and $\theta>0$.

Proof. If $X \mid \lambda \sim N B(r, p=\exp (-\lambda))$ and $\lambda \sim W G(\theta, \beta=1)$, then the pmf of $\mathrm{X}$ can easily be obtained as

$$
f(x ; r, \theta)=\frac{\theta}{(\theta+2)}\left(\begin{array}{c}
r+x-1 \\
x
\end{array}\right) \sum_{j=0}^{x}\left(\begin{array}{l}
x \\
j
\end{array}\right)(-1)^{j} \frac{\theta+(\theta+1)(\theta+r+j)}{(\theta+r+j)^{2}} .
$$

Corollary 1.2. If $\beta=2$, then the NB-WG distribution reduces to the negative binomial-size biased Garima (NB$\mathrm{SBG})$ distribution with the pmf given as

$$
f(x ; r, \theta)=\frac{\theta^{2}}{(\theta+3)}\left(\begin{array}{c}
r+x-1 \\
x
\end{array}\right) \sum_{j=0}^{x}\left(\begin{array}{l}
x \\
j
\end{array}\right)(-1)^{j} \frac{2 \theta+(\theta+1)(\theta+r+j)}{(\theta+r+j)^{3}}
$$

where $\mathrm{x}=0,1,2, \ldots$, for $r$ and $\theta>0$.

Proof. If $X \mid \lambda \sim N B(r, p=\exp (-\lambda))$ and $\lambda \sim W G(\theta, \beta=2)$, then the pmf of $X$ can be written as

$$
f(x ; r, \theta)=\frac{\theta^{2}}{(\theta+3)}\left(\begin{array}{c}
r+x-1 \\
x
\end{array}\right) \sum_{j=0}^{x}\left(\begin{array}{l}
x \\
j
\end{array}\right)(-1)^{j} \frac{2 \theta+(\theta+1)(\theta+r+j)}{(\theta+r+j)^{3}} .
$$

From Corollary 1.1 and 1.2, we can also find that the NB-G and NB-SBG distributions are presented as a new mixed NB distribution.

\section{Some Distributional Properties}

This section presents useful expansions for the pmf of the NB-WG distribution. Some basic mathematical properties are provided, particularly the factorial moments, the first four moments, variance, skewness, and kurtosis. These results are given through the following theorem and corollaries.

Theorem 2. If $X \sim N B-W G(r, \theta, \beta)$, then the factorial moment of order $\mathrm{k}$ of $\mathrm{X}$ is given by

$$
\mu_{[k]}(X)=\frac{\Gamma(r+k)}{\Gamma(r)} \sum_{j=0}^{k}\left(\begin{array}{l}
k \\
j
\end{array}\right)(-1)^{j} \frac{\theta^{\beta}[(\theta+1)(\theta-k+j) \Gamma(\beta)+\theta \Gamma(\beta+1)]}{(\theta+\beta+1)(\theta-k+j)^{\beta+1} \Gamma(\beta)} .
$$

where $\mathrm{k}=1,2, \ldots$, for $r, \theta$ and $\beta>0$.

Proof. According to Gómez-Déniz et al. (2008), the factorial moment of order $k$ of the mixed NB distribution where $p=\exp (-\lambda)$ can be derived by using the elementary function is as follows:

$$
\begin{aligned}
\mu_{[k]}(X)= & E_{\lambda}\left(\frac{\Gamma(r+k)}{\Gamma(r)} \frac{\left(1-e^{-\lambda}\right)^{k}}{e^{-\lambda k}}\right) \\
& =\frac{\Gamma(r+k)}{\Gamma(r)} E_{\lambda}\left(e^{\lambda}-1\right)^{k} .
\end{aligned}
$$

Then, we can also apply the binomial expansion for the term $\left(e^{\lambda}-1\right)^{k}$, so

$$
\begin{aligned}
\mu_{[k]}(X)= & \frac{\Gamma(r+k)}{\Gamma(r)} \sum_{j=0}^{k}\left(\begin{array}{l}
k \\
j
\end{array}\right)(-1)^{j} E_{\lambda}\left(e^{\lambda(k-j)}\right) \\
& =\frac{\Gamma(r+k)}{\Gamma(r)} \sum_{j=0}^{k}\left(\begin{array}{l}
k \\
j
\end{array}\right)(-1)^{j} M_{\lambda}(k-j) .
\end{aligned}
$$


From the mgf of the WG distribution in (4) with $t=k-j$, we put it into (13). Finally, $\mu_{[k]}(X)$ can be written as

$$
\mu_{[k]}(X)=\frac{\Gamma(r+k)}{\Gamma(r)} \sum_{j=0}^{k}\left(\begin{array}{l}
k \\
j
\end{array}\right)(-1)^{j} \frac{\theta^{\beta}[(\theta+1)(\theta-k+j) \Gamma(\beta)+\theta \Gamma(\beta+1)]}{(\theta+\beta+1)(\theta-k+j)^{\beta+1} \Gamma(\beta)} .
$$

As a result of Theorem 2, we have the following corollaries.

Corollary 2.1. If $\beta=1$, then the factorial moment of order $\mathrm{k}$ of the NB-WG distribution reduces to the factorial moment of order $\mathrm{k}$ of the NB-G distribution, which can be shown as

$$
\mu_{[k]}(X)=\frac{\Gamma(r+k)}{\Gamma(r)} \sum_{j=0}^{k}\left(\begin{array}{l}
k \\
j
\end{array}\right)(-1)^{j} \frac{\theta^{2}+\theta(\theta+1)(\theta-k+j)}{(\theta+2)(\theta-k+j)^{2}} .
$$

Proof. Substituting $\beta=1$ into (11), we obtain

$$
\mu_{[k]}(X)=\frac{\Gamma(r+k)}{\Gamma(r)} \sum_{j=0}^{k}\left(\begin{array}{l}
k \\
j
\end{array}\right)(-1)^{j} \frac{\theta^{2}+\theta(\theta+1)(\theta-k+j)}{(\theta+2)(\theta-k+j)^{2}} .
$$

Corollary 2.2. If $\beta=2$, then the factorial moment of order $\mathrm{k}$ of the NB-WG distribution reduces to the factorial moment of order $\mathrm{k}$ of the NB-SBG distribution, which is given as

$$
\mu_{[k]}(X)=\frac{\Gamma(r+k)}{\Gamma(r)} \sum_{j=0}^{k}\left(\begin{array}{l}
k \\
j
\end{array}\right)(-1)^{j} \frac{\theta^{2}[2 \theta+(\theta+1)(\theta-k+j)]}{(\theta+3)(\theta-k+j)^{3}} .
$$

Proof. Substituting $\beta=2$ into (11), we get

$$
\mu_{[k]}(X)=\frac{\Gamma(r+k)}{\Gamma(r)} \sum_{j=0}^{k}\left(\begin{array}{l}
k \\
j
\end{array}\right)(-1)^{j} \frac{\theta^{2}[2 \theta+(\theta+1)(\theta-k+j)]}{(\theta+3)(\theta-k+j)^{3}} .
$$

Based on the factorial moments of the NB-WG distribution, the first four moments, variance, skewness, and kurtosis can be expressed as follows:

$$
\begin{gathered}
E(X)=r\left[\frac{\theta^{\beta}((\theta+1)(\theta-1)+\theta \beta)}{(\theta+\beta+1)(\theta-1)^{\beta+1}}\right]-r \\
E\left(X^{2}\right)=\left(r^{2}+r\right)\left[\frac{\theta^{\beta}((\theta+1)(\theta-2)+\theta \beta)}{(\theta+\beta+1)(\theta-2)^{\beta+1}}\right]-\left(2 r^{2}+r\right)\left[\frac{\theta^{\beta}((\theta+1)(\theta-1)+\theta \beta)}{(\theta+\beta+1)(\theta-1)^{\beta+1}}\right]+r^{2}, \\
E\left(X^{3}\right)=\left(r^{3}+3 r^{2}+2 r\right)\left[\frac{\theta^{\beta}((\theta+1)(\theta-3)+\theta \beta)}{(\theta+\beta+1)(\theta-3)^{\beta+1}}\right] \\
-\left(3 r^{3}+6 r^{2}+3 r\right)\left[\frac{\theta^{\beta}((\theta+1)(\theta-2)+\theta \beta)}{(\theta+\beta+1)(\theta-2)^{\beta+1}}\right] \\
+\left(3 r^{3}+3 r^{2}+r\right)\left[\frac{\theta^{\beta}((\theta+1)(\theta-1)+\theta \beta)}{(\theta+\beta+1)(\theta-1)^{\beta+1}}\right]-r^{3} \\
E\left(X^{4}\right)=\left(r^{4}+6 r^{3}+11 r^{2}+6 r\right)\left[\frac{\theta^{\beta}((\theta+1)(\theta-4)+\theta \beta)}{(\theta+\beta+1)(\theta-4)^{\beta+1}}\right] \\
-\left(4 r^{4}+18 r^{3}+26 r^{2}+12 r\right)\left[\frac{\theta^{\beta}((\theta+1)(\theta-3)+\theta \beta)}{(\theta+\beta+1)(\theta-3)^{\beta+1}}\right] \\
+\left(6 r^{4}+18 r^{3}+19 r^{2}+7 r\right)\left[\frac{\theta^{\beta}((\theta+1)(\theta-2)+\theta \beta)}{(\theta+\beta+1)(\theta-2)^{\beta+1}}\right]
\end{gathered}
$$




$$
\begin{aligned}
& -\left(4 r^{4}+6 r^{3}+4 r^{2}+r\right)\left[\frac{\theta^{\beta}((\theta+1)(\theta-1)+\theta \beta)}{(\theta+\beta+1)(\theta-1)^{\beta+1}}\right]+r^{4} \\
& \operatorname{Var}(X)=E\left(X^{2}\right)-(E(X))^{2}, \\
& =\left(r^{2}+r\right)\left[\frac{\theta^{\beta}((\theta+1)(\theta-2)+\theta \beta)}{(\theta+\beta+1)(\theta-2)^{\beta+1}}\right]-r\left[\frac{\theta^{\beta}((\theta+1)(\theta-1)+\theta \beta)}{(\theta+\beta+1)(\theta-1)^{\beta+1}}\right] \\
& -r^{2}\left[\frac{\theta^{\beta}((\theta+1)(\theta-1)+\theta \beta)}{(\theta+\beta+1)(\theta-1)^{\beta+1}}\right]^{2}, \\
& \operatorname{Skewness}(X)=\left[E\left(X^{3}\right)-3 E\left(X^{2}\right) E(X)+2(E(X))^{3}\right] / \sigma^{3}, \\
& =\left[\left(r^{3}+3 r^{2}+2 r\right)\left[\frac{\theta^{\beta}((\theta+1)(\theta-3)+\theta \beta)}{(\theta+\beta+1)(\theta-3)^{\beta+1}}\right]\right. \\
& -\left(3 r^{2}+3 r\right)\left[\frac{\theta^{\beta}((\theta+1)(\theta-2)+\theta \beta)}{(\theta+\beta+1)(\theta-2)^{\beta+1}}\right] \\
& +r\left[\frac{\theta^{\beta}((\theta+1)(\theta-1)+\theta \beta)}{(\theta+\beta+1)(\theta-1)^{\beta+1}}\right]+3 r^{2}\left[\frac{\theta^{\beta}((\theta+1)(\theta-1)+\theta \beta)}{(\theta+\beta+1)(\theta-1)^{\beta+1}}\right]^{2} \\
& -\left(3 r^{3}+3 r^{2}\right)\left[\frac{\theta^{\beta}((\theta+1)(\theta-1)+\theta \beta)}{(\theta+\beta+1)(\theta-1)^{\beta+1}}\right]\left[\frac{\theta^{\beta}((\theta+1)(\theta-2)+\theta \beta)}{(\theta+\beta+1)(\theta-2)^{\beta+1}}\right] \\
& \left.+2 r^{3}\left[\frac{\theta^{\beta}((\theta+1)(\theta-1)+\theta \beta)}{(\theta+\beta+1)(\theta-1)^{\beta+1}}\right]^{3}\right] /(\operatorname{Var}(X))^{3 / 2} \text {. }
\end{aligned}
$$

$$
\begin{aligned}
& \operatorname{Kurtosis}(X)=\left[E\left(X^{4}\right)-4 E\left(X^{3}\right) E(X)+6 E\left(X^{2}\right)(E(X))^{2}-4 E(X)(E(X))^{3}\right. \\
& \left.+(E(X))^{4}\right] / \sigma^{4}, \\
& =\left[\left(r^{4}+6 r^{3}+11 r^{2}+6 r\right)\left[\frac{\theta^{\beta}((\theta+1)(\theta-4)+\theta \beta)}{(\theta+\beta+1)(\theta-4)^{\beta+1}}\right]\right. \\
& -\left(6 r^{3}+18 r^{2}+12 r\right)\left[\frac{\theta^{\beta}((\theta+1)(\theta-3)+\theta \beta)}{(\theta+\beta+1)(\theta-3)^{\beta+1}}\right] \\
& +\left(7 r^{2}+7 r\right)\left[\frac{\theta^{\beta}((\theta+1)(\theta-2)+\theta \beta)}{(\theta+\beta+1)(\theta-2)^{\beta+1}}\right] \\
& -r\left[\frac{\theta^{\beta}((\theta+1)(\theta-1)+\theta \beta)}{(\theta+\beta+1)(\theta-1)^{\beta+1}}\right] \\
& -4 r^{2}\left[\frac{\theta^{\beta}((\theta+1)(\theta-1)+\theta \beta)}{(\theta+\beta+1)(\theta-1)^{\beta+1}}\right]^{2} \\
& +\left(12 r^{3}+12 r^{2}\right)\left[\frac{\theta^{\beta}((\theta+1)(\theta-1)+\theta \beta)}{(\theta+\beta+1)(\theta-1)^{\beta+1}}\right]\left[\frac{\theta^{\beta}((\theta+1)(\theta-2)+\theta \beta)}{(\theta+\beta+1)(\theta-2)^{\beta+1}}\right] \\
& -\left(4 r^{4}+12 r^{3}+8 r^{2}\right)\left[\frac{\theta^{\beta}((\theta+1)(\theta-1)+\theta \beta)}{(\theta+\beta+1)(\theta-1)^{\beta+1}}\right]\left[\frac{\theta^{\beta}((\theta+1)(\theta-3)+\theta \beta)}{(\theta+\beta+1)(\theta-3)^{\beta+1}}\right] \\
& +\left(6 r^{4}+6 r^{3}\right)\left[\frac{\theta^{\beta}((\theta+1)(\theta-1)+\theta \beta)}{(\theta+\beta+1)(\theta-1)^{\beta+1}}\right]^{2}\left[\frac{\theta^{\beta}((\theta+1)(\theta-2)+\theta \beta)}{(\theta+\beta+1)(\theta-2)^{\beta+1}}\right] \\
& -6 r^{3}\left[\frac{\theta^{\beta}((\theta+1)(\theta-1)+\theta \beta)}{(\theta+\beta+1)(\theta-1)^{\beta+1}}\right]^{3} \\
& \left.-3 r^{4}\left[\frac{\theta^{\beta}((\theta+1)(\theta-1)+\theta \beta)}{(\theta+\beta+1)(\theta-1)^{\beta+1}}\right]^{4}\right] /(\operatorname{Var}(X))^{2} \text {. }
\end{aligned}
$$




\section{Estimation of Parameters}

In this section, the parameter estimation of $N B-W G(r, \theta, \beta)$ will be done via Maximum Likelihood Estimation (MLE) procedure.

Consider $\left(x_{1}, x_{2}, \ldots, x_{n}\right)$ is a random sample of size $n$ from the $N B-W G$ distribution in (5). The likelihood function of the $N B-W G(r, \theta, \beta)$ is given by

$$
L(r, \theta, \beta)=\prod_{i=1}^{n} \frac{\theta^{\beta}}{(\theta+\beta+1) \Gamma(\beta)}\left(\begin{array}{c}
r+x_{i}-1 \\
x_{i}
\end{array}\right) \sum_{j=0}^{x_{i}}\left(\begin{array}{c}
x_{i} \\
j
\end{array}\right)(-1)^{j} \frac{(\theta+1)(\theta+r+j) \Gamma(\beta)+\theta \Gamma(\beta+1)}{(\theta+r+j)^{\beta+1}}
$$

then the log-likelihood function can be calculated as

$$
\begin{array}{r}
\log L(r, \theta, \beta)=\sum_{i=1}^{n}\left[\log \left(r+x_{i}-1\right) !-\log x_{i} !-\log (r-1) !\right]+n \beta \log (\theta)-n \log (\theta+\beta+1) \\
-n \log \Gamma(\beta)+\sum_{i=1}^{n}\left[\log \sum_{j=0}^{x_{i}}\left(\begin{array}{c}
x_{i} \\
j
\end{array}\right)(-1)^{j} \frac{(\theta+1)(\theta+r+j) \Gamma(\beta)+\theta \Gamma(\beta+1)}{(\theta+r+j)^{\beta+1}}\right] .
\end{array}
$$

The maximum likelihood estimates $(\hat{r}, \hat{\theta}, \hat{\beta})$ of $(r, \theta, \beta)$ come from the solution of the following partial differential equations:

$$
\begin{aligned}
& \frac{\partial}{\partial r} \log L(r, \theta, \beta)=\sum_{i=1}^{n}\left(\frac{\frac{\partial}{\partial r} \Gamma\left(r+x_{i}\right)}{\Gamma\left(r+x_{i}\right)}-\frac{\frac{\partial}{\partial r} \Gamma(r)}{\Gamma(r)}\right) \\
& +\sum_{i=1}^{n} \frac{\partial}{\partial r} \log \sum_{j=0}^{x_{i}}\left(\begin{array}{c}
x_{i} \\
j
\end{array}\right)(-1)^{j} \frac{(\theta+1) w_{j} \Gamma(\beta)+\theta \Gamma(\beta+1)}{w_{j}^{\beta+1}} \\
& =\sum_{i=1}^{n} \psi\left(r+x_{i}\right)-n \psi(r)+\sum_{i=1}^{n}\left(\frac{\sum_{j=0}^{x_{i}}\left(\begin{array}{c}
x_{i} \\
j
\end{array}\right)(-1)^{j} \frac{\partial}{\partial r} \frac{(\theta+1) w_{j} \Gamma(\beta)+\theta \Gamma(\beta+1)}{w_{j}^{\beta+1}}}{\sum_{j=0}^{x_{i}}\left(\begin{array}{c}
x_{i} \\
j
\end{array}\right)(-1)^{j} \frac{(\theta+1) w_{j} \Gamma(\beta)+\theta \Gamma(\beta+1)}{w_{j}^{\beta+1}}}\right) \\
& =\sum_{i=1}^{n} \psi\left(r+x_{i}\right)-n \psi(r) \\
& +\sum_{i=1}^{n}\left(\frac{\sum_{j=0}^{x_{i}}\left(\begin{array}{c}
x_{i} \\
j
\end{array}\right)(-1)^{j}\left\{(\theta+1) w_{j}^{-\beta-1} \Gamma(\beta)-(\beta+1) w_{j}^{-\beta-2}\left[(\theta+1) w_{j} \Gamma(\beta)+\theta \Gamma(\beta+1)\right]\right\}}{\sum_{j=0}^{x_{i}}\left(\begin{array}{c}
x_{i} \\
j
\end{array}\right)(-1)^{j} \frac{(\theta+1) w_{j} \Gamma(\beta)+\theta \Gamma(\beta+1)}{w_{j}^{\beta+1}}}\right) \text {, } \\
& \frac{\partial}{\partial \theta} \log L(r, \theta, \beta) \\
& =n \beta \frac{\partial}{\partial \theta} \log (\theta)-n \frac{\partial}{\partial \theta} \log (\theta+\beta+1) \\
& +\sum_{i=1}^{n} \frac{\partial}{\partial \theta} \log \sum_{j=0}^{x_{i}}\left(\begin{array}{c}
x_{i} \\
j
\end{array}\right)(-1)^{j} \frac{(\theta+1) w_{j} \Gamma(\beta)+\theta \Gamma(\beta+1)}{w_{j}{ }^{\beta+1}} \\
& =\frac{n \beta}{\theta}-\frac{n}{\theta+\beta+1}
\end{aligned}
$$




$$
\begin{aligned}
& +\sum_{i=1}^{n}\left(\frac{\sum_{j=0}^{x_{i}}\left(\begin{array}{c}
x_{i} \\
j
\end{array}\right)(-1)^{j}\left\{w_{j}^{-\beta-1}\left[w_{j} \Gamma(\beta)+(\theta+1) \Gamma(\beta)+\Gamma(\beta+1)\right]\right.}{\sum_{j=0}^{x_{i}}\left(\begin{array}{c}
x_{i} \\
j
\end{array}\right)(-1)^{j} \frac{(\theta+1) w_{j} \Gamma(\beta)+\theta \Gamma(\beta+1)}{w_{j}^{\beta+1}}}\right. \\
& -\frac{\left.(\beta+1) w_{j}^{-\beta-2}\left[(\theta+1) w_{j} \Gamma(\beta)+\theta \Gamma(\beta+1)\right]\right\}}{\left.\sum_{j=0}^{x_{i}}\left(\begin{array}{c}
x_{i} \\
j
\end{array}\right)(-1)^{j} \frac{(\theta+1) w_{j} \Gamma(\beta)+\theta \Gamma(\beta+1)}{w_{j} \beta^{\beta+1}}\right),} \\
& \frac{\partial}{\partial \beta} \log L(r, \theta, \beta) \\
& =n \log (\theta) \frac{\partial}{\partial \beta} \beta-n \frac{\partial}{\partial \beta} \log (\theta+\beta+1)-n \frac{\partial}{\partial \beta} \log \Gamma(\beta) \\
& +\sum_{i=1}^{n} \frac{\partial}{\partial \beta} \log \sum_{j=0}^{x_{i}}\left(\begin{array}{c}
x_{i} \\
j
\end{array}\right)(-1)^{j} \frac{(\theta+1) w_{j} \Gamma(\beta)+\theta \Gamma(\beta+1)}{w_{j}^{\beta+1}} \\
& =n \log (\theta)-\frac{n}{\theta+\beta+1}-n \psi(\beta) \\
& +\sum_{i=1}^{n}\left(\frac{\sum_{j=0}^{x_{i}}\left(\begin{array}{c}
x_{i} \\
j
\end{array}\right)(-1)^{j}\left\{w_{j}^{-\beta-1}\left[(\theta+1) w_{j} \Gamma(\beta) \psi(\beta)+\theta \Gamma(\beta+1) \psi(\beta+1)\right]\right.}{\sum_{j=0}^{x_{i}}\left(\begin{array}{c}
x_{i} \\
j
\end{array}\right)(-1)^{j} \frac{(\theta+1) w_{j} \Gamma(\beta)+\theta \Gamma(\beta+1)}{w_{j}^{\beta+1}}}\right. \\
& \left.-\frac{\left.w_{j}^{-z-1}\left[(\theta+1) w_{j} \Gamma(\beta)+\theta \Gamma(\beta+1)\right] \log w_{j}\right\}}{\sum_{j=0}^{x_{i}}\left(\begin{array}{c}
x_{i} \\
j
\end{array}\right)(-1)^{j} \frac{(\theta+1) w_{j} \Gamma(\beta)+\theta \Gamma(\beta+1)}{w_{j}^{\beta+1}}}\right),
\end{aligned}
$$

where $w_{j}=r+\theta+j$.

With the above equating to zero, these three equations cannot be solved analytically because they are not in closed forms. However, we can obtain the MLE solution of $(\hat{r}, \hat{\theta}, \hat{\beta})$ by using the nonlinear minimization (nlm) function in the statistical package of $\mathrm{R}$ language (R Core Team, 2018). This nlm function implements the Newton-Raphson method for minimizing a function given a vector of starting values.

\section{Results and Discussion}

In this section, we discuss applications of the NB-WG to real data. The first data set, obtained from Pudprommarat et al. (2012), provide information about the number of injured in an accident on a major road in Bangkok, Thailand in 2007. As shown in Table 1, the data contain evidence of overdispersion with a mean $=0.3456$, variance $=0.6556$ and index of dispersion $=1.8969$. Additionally, another application of the NB-WG is the number of hospital stays of United States' residents aged 66 and over (see Flynn, 2009), which is displayed in Table 2. It has overdispersion with an index of dispersion equal to 1.8824 .

In order to compare distributions, we apply the Poisson, NB and NB-WG distributions to fit these two real data sets. The distribution parameters are estimated by the MLE. The best distribution is the distribution that corresponds to lower values of negative log-likelihood and AIC (Akaike Information Criterion), and a higher p-value of chi-square from a goodness of fit test. Based on the results in Table 1-2, we conclude that the NB-WG distribution is competing well against the others and gives a satisfactory fit. 
Table 1: Observed and expected frequencies for the roadway accident data

\begin{tabular}{|c|c|c|c|c|}
\hline \multirow{2}{*}{ No. of injured } & \multirow{2}{*}{ Observed } & \multicolumn{3}{|c|}{ Fitting distributions } \\
\hline & & Poisson & NB & NB-WG \\
\hline 0 & 1273 & 1187.6 & 1278.7 & 1278.3 \\
\hline 1 & 300 & 410.5 & 278.1 & 286.1 \\
\hline 2 & 71 & 70.9 & 81.8 & 75.9 \\
\hline 3 & 18 & & 26.2 & 23.6 \\
\hline 4 & 9 & $\zeta_{89}$ & & 8.3 \\
\hline 5 & 4 & 8.9 & 13.2 & $\{5.9$ \\
\hline \multirow{2}{*}{\multicolumn{2}{|c|}{ Estimated parameters }} & $\hat{\lambda}=0.346$ & $\hat{r}=0.587$ & $\hat{r}=1.729$ \\
\hline & & & $\hat{p}=0.629$ & $\begin{array}{l}\hat{\theta}=10.018 \\
\hat{\beta}=1.592\end{array}$ \\
\hline \multirow{2}{*}{\multicolumn{2}{|c|}{$\begin{array}{l}\text {-Log likelihood } \\
\text { AIC }\end{array}$}} & 1368.373 & 1279.830 & 1274.125 \\
\hline & & 2738.746 & 2563.660 & 2554.250 \\
\hline \multirow{3}{*}{\multicolumn{2}{|c|}{$\begin{array}{l}\text { Chi-squares } \\
\text { Degree of freedom } \\
p \text {-value }\end{array}$}} & 106.236 & 6.301 & 2.598 \\
\hline & & 2 & 2 & 2 \\
\hline & & $<0.0001$ & 0.0428 & 0.2728 \\
\hline
\end{tabular}

Table 2: Observed and expected frequencies of the number of hospital stays of United States' residents aged 66 and over

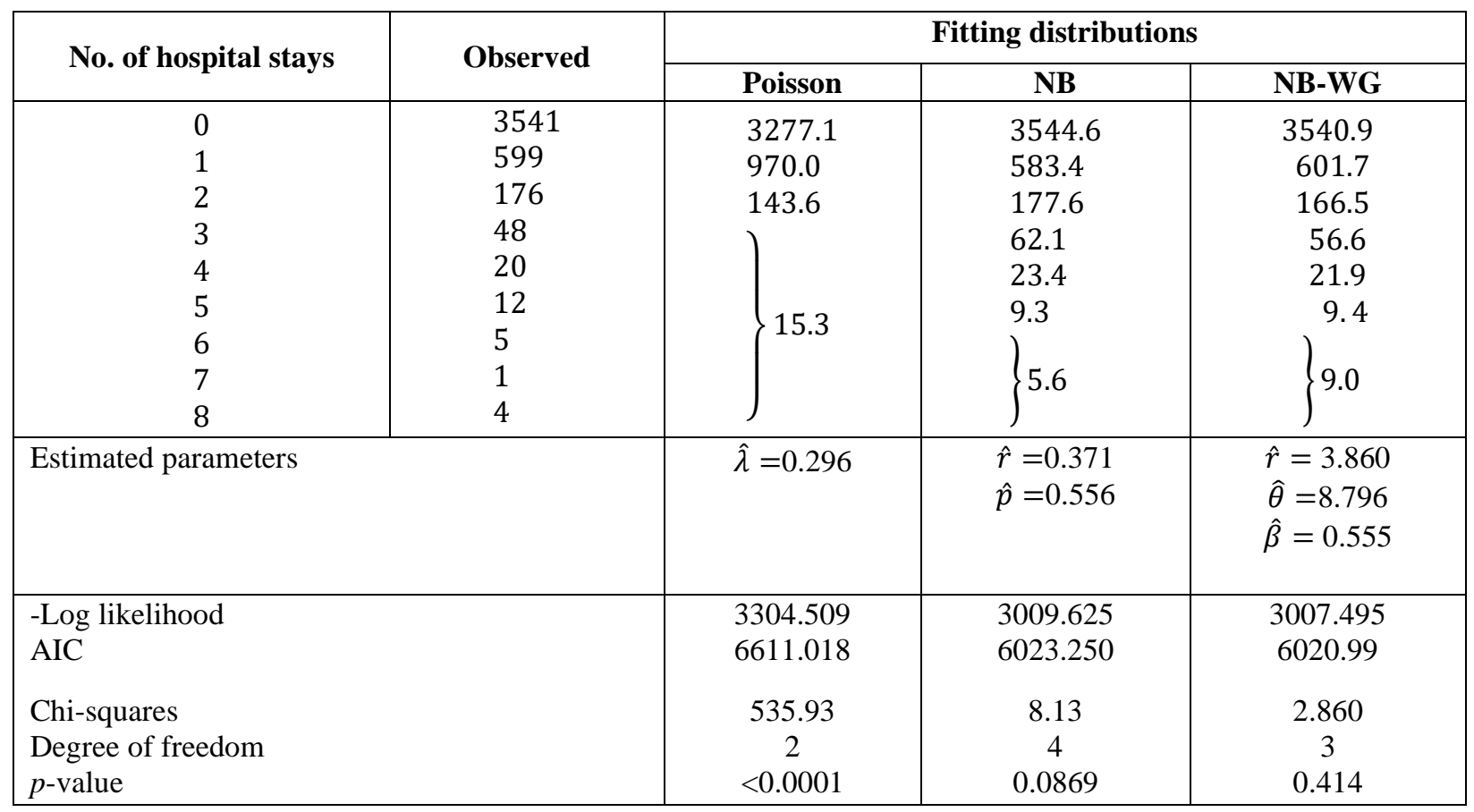

\section{Conclusions}

This paper presents the NB-WG distribution which is obtained by mixing the NB distribution with a two-parameter weighted Garima distribution. We find that the NB-G and NB-SBG distributions are all special cases of the NB-WG distribution and they both can also be considered as the new mixed NB distributions. The factorial moments, the first four moments, variance, skewness, and kurtosis of the proposed distribution have been discussed. In addition, the parameter estimation is carried out via the method of maximum likelihood estimation. Finally, two real data sets have 
been considered for the goodness of fit test. The results show that the NB-WG distribution provides the best fit of both data sets, and we expect this distribution can serve as an alternative approach to modeling over-dispersed count data.

\section{References}

1. Balakrishnan, N. and Nevzorov, V.B. (2003). A Primer on Statistical Distributions. John Wiley. New York.

2. Eyob, T. and Shanker, R. (2018). A two-parameter weighted Garima distribution with properties and application. Biometrics \& Biostatistics International Journal, 7, 234-242.

3. Flynn, M. (2009). More flexible GLMs zero-inflated models and hybrid models. Casualty Actuarial Society E-Forum, Winter, 148-224.

4. Gómez-Déniz, E., Sarabia, J. M. and Calderín-Ojeda, E. (2008). Univariate and multivariate versions of the negative binomial - inverse Gaussian distributions with applications. Insurance: Mathematics and Economics, 42, 39-49.

5. Kongrod, S., Bodhisuwan, W. and Payakkapong, P. (2014). The negative binomial- Erlang distribution with applications. International Journal of Pure and Applied Mathematics, 92, 389-401.

6. Lord, D. and Geedipally, S. R. (2011). The negative binomial-Lindley distribution as a tool for analysing crash data characterized by a large amount of zeros. Accident Analysis \& Prevention, 43, 1738-1742.

7. Pudprommarat, C., Bodhisuwan, W. and Zeephongsekul, P. (2012). A new mixed negative binomial distribution. Journal of Applied Sciences, 12, 1853-1858.

8. R Core Team. (2018). R: A Language and Environment for Statistical Computing. R Foundation for Statistical Computing. Vienna, Austria. https://www.R-project.org/.

9. Rainer, W. (2008). Econometric Analysis of Count Data. SpringerVerlag. Germany.

10. Willmot, G.E. (1987). The Poisson-inverse Gaussian distribution as an alternative to the negative binomial. Scandinavian Actuarial Journal, 113-127.

11. Yamrubboon, D., Bodhisuwan, W., Pudprommarat, C., and Saothayanun, L. (2017). The negative binomialSushila distribution with application in count data analysis. Thailand Statistician, 15, 69-77.

12. Zamani, H. and Ismail, N. (2010). Negative binomial Lindley distribution and its application. Journal Mathematics and Statistics, 6, 4-9. 